\title{
Efficient Planning Tool for WDM Transport Networks
}

\author{
C. BUNGARZEANU, L. BESUCHET, D. RODELLAR \\ Ecole Polytechnique Fédérale de Lausanne \\ DE - Laboratoire de Télécommunications \\ CH-1015 Lausanne, Switzerland \\ E-maiL:Cristian.Bungarzeanu@epfl.ch
}

Key words: WDM, all optical networks, wavelength assignment, network planing

\begin{abstract}
Last generation optical components jointly with WDM techniques will allow the realisation of a switched optical layer based on wavelength routing of semi-permanent paths. This paper presents a computer-aided planning tool intended to cover the dimensioning, routing, and wavelength assignment in meshed networks with WDM optical cross-connects. An efficient graphcolouring algorithm allows a fast wavelength assignment even for topologies with a large number of nodes. The performances of the planning tool are illustrated on a 8-nodes European optical network.
\end{abstract}

\section{INTRODUCTION}

In response to increasing pressure on transmission capacity, brought about by upsurge in both narrow-band and emerging broadband services, many carriers are considering deploying dense wavelength-division multiplex (WDM) systems as a means of taking advantage of the fibre bandwidth [1]. In addition to point-to-point line systems, new optical elements with networking features, such as optical add/drop multiplexers (OADM) and optical cross-connects (OXC) have been tested in field trials. 
These optical components of last generation allow for the manipulation of wavelengths in WDM networks offering advanced features such as wavelength routing, switching, and conversion/interchange [2]. In this way, protocol-transparent optical channels can be implemented between any source-destination nodes. Moreover, WDM techniques offer enough spare capacity for protection and restoration purposes. The design of WDM networks, including topics such as topology optimisation, routing and dimensioning, wavelength assignment, protection and restoration represents a complex optimisation problem.

In this context, there is a growing need for planning tools to deal with the main issues of optical networking in an automated manner. This paper describes a software application intended to address the planning of highspeed WDM networks. This software tool is currently under development so that several major planning issues (protection and restoration, transmission quality) will be dealt with in the near future.

\section{NETWORK MODELLING}

Nodes consist of optical cross-connects with the architecture depicted in Fig. 1. The number of output fibres $M$ is the same as the number of input fibres. Each fibre carries $L$ wavelengths. The optically transparent OXC is able to connect any wavelength from an incoming fibre to the corresponding wavelength of one of the outgoing fibre. It is assumed that there is no wavelength conversion within the $\mathrm{OXC}$, so that there is no need of wavelength converters and the network management is simplified since only one wavelength is attached to an end-to-end channel. However, a blocking situation may occur if two channels having the same wavelength attempt to use the same outgoing fibre. Each OXC performs a static routing, switching occurring only in case a reconfiguration after a failure. 


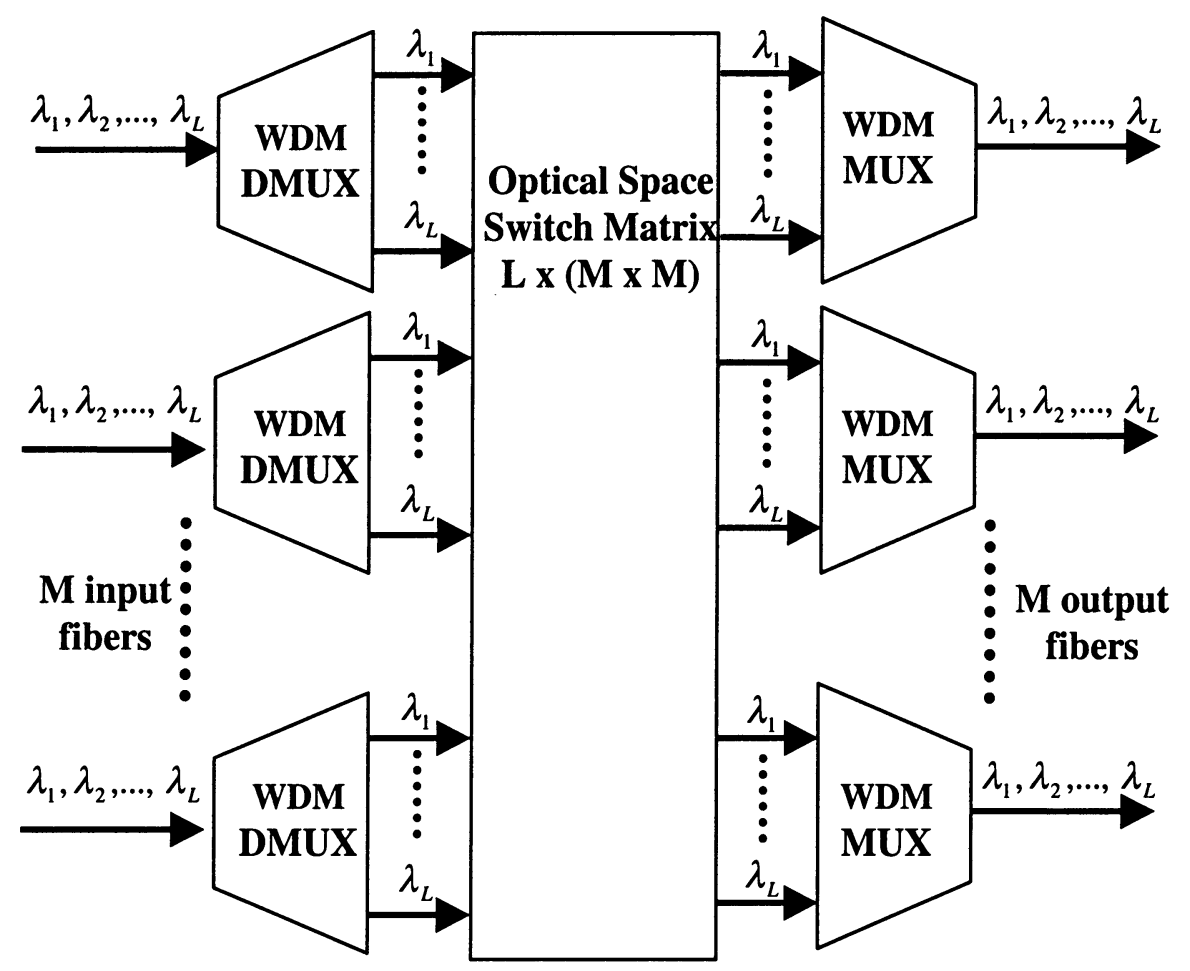

Fig. 1 Optical cross-connect

As shown in Fig. 2., a link $l$ contains $k$ fibres, each fibre supporting up to $L$ wavelengths ( $L$ is constant over the network). Thus, the capacity of the link $l$, expressed in terms of optical channels is $C_{l}=k L$.

\subsection{Routing and fibre assignment}

Routing can be performed manually or automatically. A shortest path routing is available by running Floyd's algorithm [3], which computes simultaneously the routes for all connections. Two options are available:

- minimise the connection length

- minimise the number of intermediate nodes 


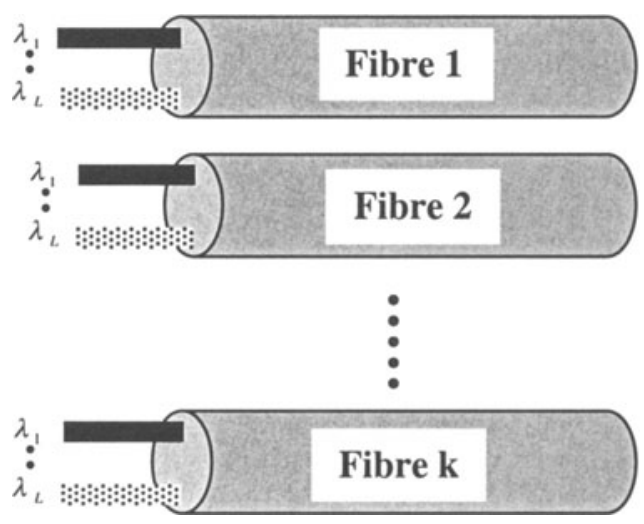

Fig. 2 Link structure

An automated fibre assignment is done for each link in the following way: the first channel is assigned to the first fibre, the second channel is assigned to the second fibre, the $k$ channel is assigned to the $k$ fibre, the $k+1$ channel is assigned to the first fibre and so on. In this way, the channels sharing the same link are equally distributed over the available fibres of the link. If the number of channels using a particular link exceeds the link capacity a warning message is issued to increase the number of fibres. At this stage, the wavelength assignment is not yet performed and even though the link capacity is greater than the number of channels that share it, it could be not sufficient to allow a global wavelength assignment solution. If, because of wavelength assignment restrictions, new fibres are added, the channels are redistributed to ensure an equal load of each fibre.

\subsection{Wavelength assignment}

The wavelength assignment is reduced to a graph colouring problem [4][5]. Each end-to-end channel is represented by a vertex in a neighbourhood graph. Vertices are connected by an edge if and only if the corresponding channels share some common fibre. The wavelength allocation can be mapped to a graph node colouring problem, which is a well-known NP complete problem. The heuristic method employed in our software is based on the Tabu search technique called TABUCOL [6]. Tabu search is a global search technique that moves step by step towards the minimum value of a function, which is in our case the number of neighbours (vertices connected by an edge) having the same colour. A tabu list of forbidden movements is updated during the iterations to avoid cycling and 
being trapped in local minima. In [6] it is shown that for graph colouring, TABUCOL is significantly superior to the simulated annealing.

The performance of the wavelength allocation technique was estimated on the network architecture shown in Fig. 3.

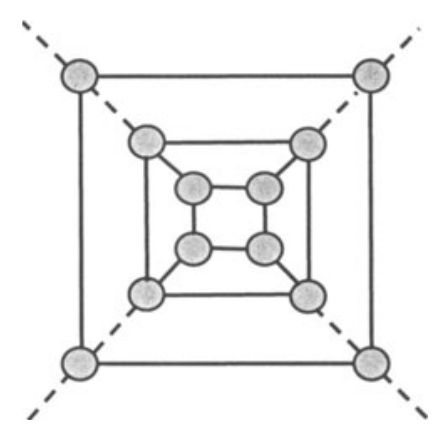

Fig. 3 Spider web test architecture

Assuming for simplicity only one fibre per link and one channel between each node pair, it turned out that the number of wavelengths per fibre $\mathrm{L}$ depends linearly on the number of node (see Fig. 4). However, as it is shown in Fig. 5, the number of iterations necessary to get a colouring solution grows exponentially.

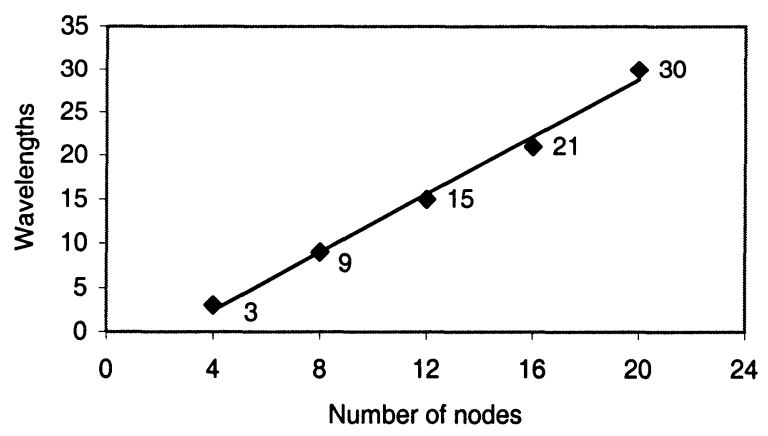

Fig. 4 Minimum number of wavelengths versus the number of nodes for the web network architecture 


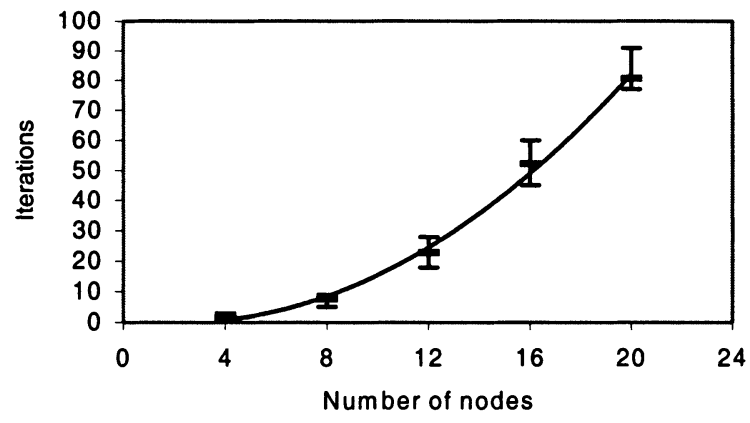

Fig. 5 Number of iterations necessary for a colouring solution

\section{EUROPEAN TRANSPORT NETWORK}

The planning tool described above has been used to design a 8-nodes central European network [7] the topology shown in Fig. 6. The distances between nodes and the demands expressed in terms of optical channels are listed in Table 1.

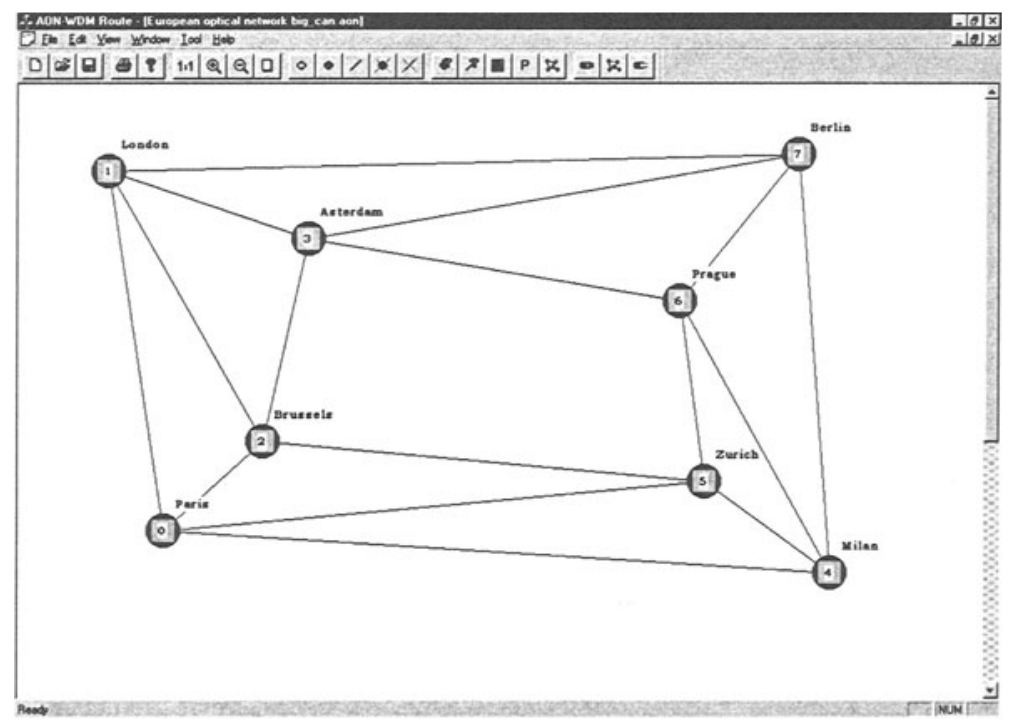

Fig. 6 European transport network topology 
Table 1

\begin{tabular}{|c|c|c|}
\hline Node pair & $\begin{array}{c}\text { Distance } \\
{[\mathbf{k m}]}\end{array}$ & $\begin{array}{c}\text { Demand } \\
\text { [channels] }\end{array}$ \\
\hline $0-1$ & 480 & 36 \\
$0-2$ & 300 & 28 \\
$0-3$ & 560 & 12 \\
$0-4$ & 740 & 28 \\
$0-5$ & 640 & 32 \\
$0-6$ & 780 & 4 \\
$0-7$ & 1160 & 40 \\
$1-2$ & 320 & 12 \\
$1-3$ & 360 & 20 \\
$1-4$ & 1040 & 16 \\
$1-5$ & 860 & 12 \\
$1-6$ & 840 & 4 \\
$1-7$ & 1040 & 36 \\
$2-3$ & 260 & 24 \\
$2-4$ & 740 & 8 \\
$2-5$ & 540 & 4 \\
$2-6$ & 580 & 4 \\
$2-7$ & 880 & 16 \\
$3-4$ & 820 & 8 \\
$3-5$ & 620 & 8 \\
$3-6$ & 480 & 4 \\
$3-7$ & 700 & 32 \\
$4-5$ & 220 & 24 \\
$4-6$ & 500 & 4 \\
$4-7$ & 900 & 36 \\
$5-6$ & 320 & 4 \\
$5-7$ & 740 & 44 \\
$6-7$ & 420 & 8 \\
\hline
\end{tabular}

The goal of the planning process was to determine the minimum number of fibres for each link, which allows a wavelength allocation solution. In this way, $1,4,8$ or 16 wavelengths per fibre have been considered. The results are listed in Table 2.

Table 2

\begin{tabular}{|c|c|c|c|r|}
\hline & \multirow{2}{*}{ Link load } & \multicolumn{3}{|c|}{ Fibres/link } \\
\cline { 3 - 5 } Link & [channels] & $\mathbf{4 \lambda}$ & $\mathbf{8} \boldsymbol{\lambda}$ & $\mathbf{1 6} \boldsymbol{\lambda}$ \\
\hline $0-1$ & 36 & 18 & 12 & 8 \\
$0-2$ & 80 & 40 & 23 & 15 \\
$0-4$ & 28 & 14 & 10 & 4 \\
$0-5$ & 36 & 17 & 11 & 7 \\
$1-2$ & 40 & 18 & 11 & 7 \\
$1-3$ & 24 & 11 & 10 & 4 \\
$1-7$ & 36 & 14 & 9 & 6 \\
$2-3$ & 104 & 48 & 33 & 19 \\
$2-5$ & 48 & 19 & 14 & 10 \\
$3-6$ & 20 & 10 & 8 & 3 \\
$3-7$ & 88 & 40 & 26 & 15 \\
$4-5$ & 48 & 17 & 10 & 7 \\
$4-6$ & 12 & 7 & 4 & 2 \\
$4-7$ & 36 & 15 & 9 & 5 \\
$5-6$ & 52 & 21 & 11 & 9 \\
$6-7$ & 52 & 23 & 13 & 7 \\
\hline
\end{tabular}


The capacity utilisation is defined as the ratio $l /(k L)$, where $l$ is the link load, $k$ is the number of fibres and $L$ is number of wavelengths per fibre.

Fig. 7 displays the capacity utilisation for each link of the 8-nodes European network. It turns out that the capacity utilisation decreases when the number of wavelengths per fibre gets higher. This is due to the increase in the number of edges in the neighbourhood graph since a higher amount of channels share the same fibre. Anyway, as it is shown in Fig. 8, the use of higher number of wavelengths allows a substantial decrease of the total amount of fibre.

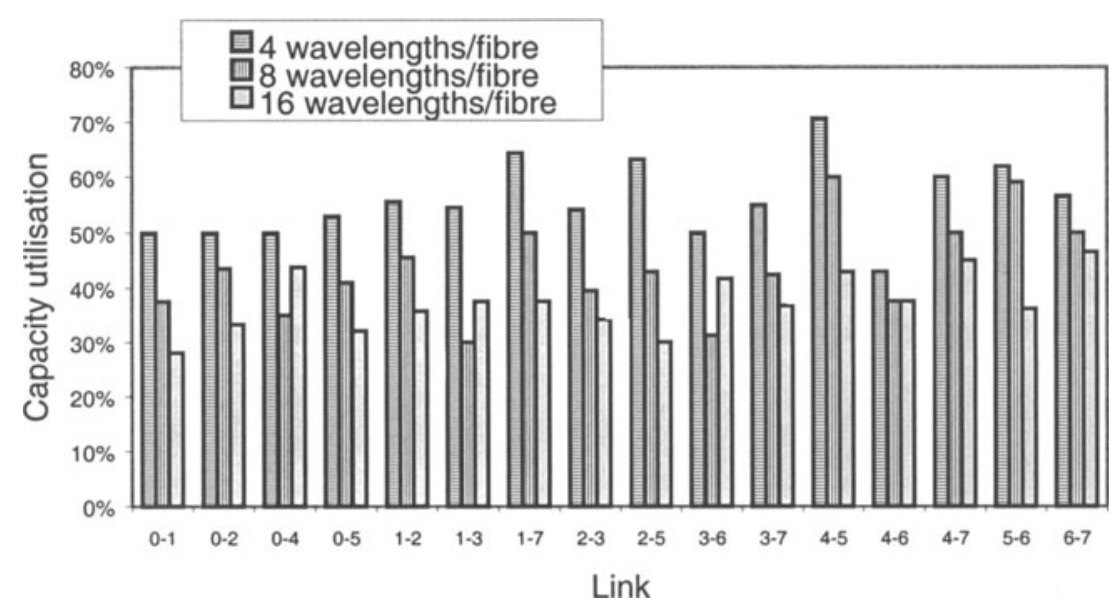

Fig. 7 Capacity utilisation

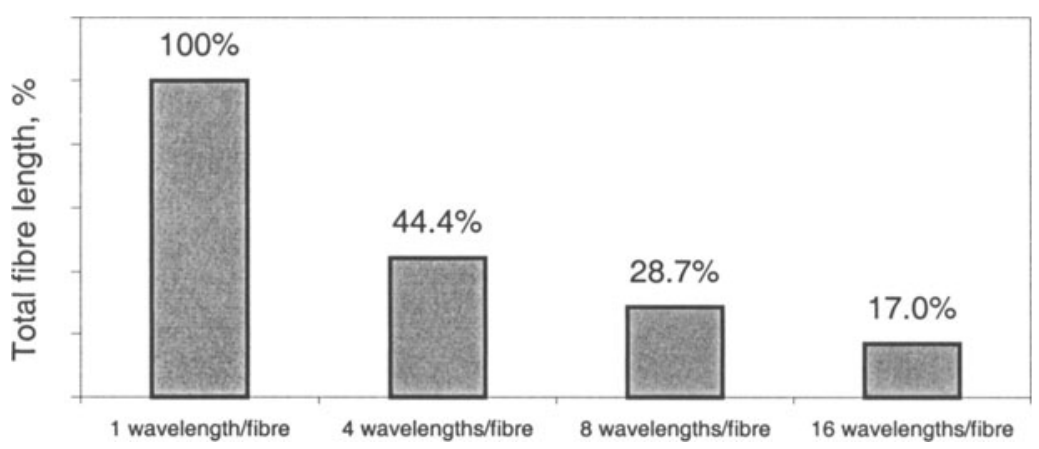

Fig. 8 Relative overall fibre length 


\section{CONCLUSION}

The software tool described above performs a fast evaluation of an existing network or a new architecture under different load situations. Various "what if" scenarios can be tested allowing the planners to select the most suitable alternative. In the near future, this planning tool will be further developed to address key issues such as protection and quality of transmission.

\section{ACKNOWLEDGEMENT}

This work has been done within the COST Action 266 "Advanced infrastructure for photonic networks"

\section{REFERENCES}

[1] E. Lowe, "Current European WDM deployment trends," IEEE Communications Magazine, vol. 36, nr. 2, February 1998.

[2] A. McGuire and P. Bonenfant, "Standards: the blueprints for optical networking," IEEE Communications Magazine, vol. 36, nr. 2, February 1998.

[3] A. Kershenbaum, Telecommunications network design algorithms, McGraw Hill 1993.

[4] T.K. Tan and J.K. Pollard, "Determination of minimum number of wavelengths required for all-optical WDM networks using graph colouring" Electronics letters, vol. 31, nr. 22, February 1995.

[5] L. Besuchet, Routage optimal et assignation des longueurs d'onde dans un réseau de communications optiques WDM, Projet de diplôme 1060 EPF-Lausanne DE-TCOM 1999.

[6] A. Herz and D. de Wera, "Using Tabu search techniques for graph colouring" Computing, vol. 39, Springer-Verlag 1987.

[7] C. Bungarzeanu and A. Colarusso, "European optical transport network" Proc. COST Workshop 94: Optical telecommunications, Nice, April 17-21, 1994. 